\title{
Physical constraints on the likelihood of life on exoplanets
}

\author{
Manasvi Lingam' and Abraham Loeb ${ }^{2}$ \\ ${ }^{1}$ John A. Paulson School of Engineering and Applied Sciences, Harvard University, 29 Oxford St, Cambridge, MA 02138, \\ USA e-mail: manasvi.lingam@cfa.harvard.edu \\ ${ }^{2}$ Harvard-Smithsonian Center for Astrophysics, 60 Garden St, Cambridge, MA 02138, USA
}

\begin{abstract}
One of the most fundamental questions in exoplanetology is to determine whether a given planet is habitable. We estimate the relative likelihood of a planet's propensity towards habitability by considering key physical characteristics such as the role of temperature on ecological and evolutionary processes, and atmospheric losses via hydrodynamic escape and stellar wind erosion. From our analysis, we demonstrate that Earth-sized exoplanets in the habitable zone around M-dwarfs seemingly display much lower prospects of being habitable relative to Earth, owing to the higher incident ultraviolet fluxes and closer distances to the host star. We illustrate our results by specifically computing the likelihood (of supporting life) for the recently discovered exoplanets, Proxima $b$ and TRAPPIST-1e, which we find to be several orders of magnitude smaller than that of Earth.

Received 13 April 2017, accepted 30 May 2017, first published online 6 July 2017
\end{abstract}

Key words: atmospheric escape, biodiversity, exoplanets, habitability, likelihood of life, metabolic theory of ecology.

\section{Introduction}

The field of exoplanetary science has witnessed many rapid and exciting advances over the past couple of decades. With the number of discovered exoplanets now numbering in the thousands (Winn \& Fabrycky 2015), there has been a great deal of interest in identifying planets that are habitable, i.e. potentially capable of bearing life (Kasting \& Catling 2003; Chyba \& Hand 2005; Lammer et al. 2009; Cockell et al. 2016). To this end, most of the current discoveries have been centered around planets orbiting $M$-dwarfs since they are more abundant, and easier to detect (Scalo et al. 2007; Tarter et al. 2007; Shields et al. 2016). It is now believed that there are $\sim 10^{10}$ habitable planets in our own Galaxy (Kasting et al. 2014; Dressing \& Charbonneau 2015).

In recent times, several major discoveries have provided additional impetus to studies of habitability. The first was the discovery of an Earth-mass exoplanet in the habitable zone (HZ) - the region capable of sustaining liquid water on the planet's surface - around Proxima Centauri, the nearest star to our Solar System at only 4.2 light years away (Anglada-Escudé et al. 2016). There are plans already underway to explore this planet, dubbed Proxima $b$, via a flyby mission. ${ }^{1}$ The second major advance was the discovery of at least seven Earth-sized planets transiting the ultracool dwarf star TRAPPIST-1 at a distance of 39.5 light years (Gillon et al. 2016, 2017). The presence of three planets in the $\mathrm{HZ}$ around

\footnotetext{
${ }^{1}$ https://breakthroughinitiatives.org/Initiative/3
}

TRAPPIST-1 therefore presents a unique opportunity for studying multiple planets that may host life (Lingam \& Loeb 2017). The discovery of the temperate super-Earth LHS $1140 \mathrm{~b}$ transiting an $M$-dwarf at a distance of 39 light years also merits a mention (Dittmann et al. 2017).

It is worth emphasizing that the existence of a planet in the $\mathrm{HZ}$ does not imply life exists on the planet, or even that it will necessarily be able to support a biosphere. Most of the current assessments of habitable planets have tended to focus on superficial metrics, which can be misleading, as rightly pointed out in Schulze-Makuch \& Guinan (2016) and Tasker et al. (2017). These metrics evaluate the degree of similarity between certain, physically relevant, parameters of a given exoplanet and the Earth, and have led to unfortunate misconceptions that planets with higher values (of the similarity indices) are automatically more habitable.

In this paper, we attempt to advance the assessment of lifebearing planets by identifying physical processes that play a major role in governing habitability through the formulation of likelihood functions that depend on basic planetary and stellar parameters. By doing so, we expect to pave the way towards understanding the complex relationship between the aforementioned parameters and the likelihood of a planet hosting life in reality (Lineweaver \& Chopra 2012; Cockell et al. 2016).

\section{The role of planetary temperature}

We begin by outlining the centrality of temperature in regulating a diverse array of ecological and evolutionary parameters and processes (Cossins \& Bowler 1987; Brown et al. 2004; Deutsch et al. 2008; Kingsolver \& Huey 2008; Angilletta 2009; Dillon et al. 2010) by adopting the general premise 
that biochemical reactions analogous to metabolism are universal on life-bearing exoplanets (Pace 2001; Benner et al. 2004; Ball 2008). Subsequently, we construct a global likelihood function for these processes that depends on the planetary temperature.

\section{The metabolic theory of ecology and temperature}

We adopt the tenets of the metabolic theory of ecology (MTE) that relies on the assumption that the metabolic rate $B$ of organisms plays a major role in governing macroecological processes. The reader may consult West et al. (2001); Agutter \& Wheatley (2004); Brown et al. (2004); Clarke \& Fraser (2004); Marquet et al. (2005); Clarke (2006); Savage et al. (2008); Price et al. (2012); Humphries \& McCann (2014); Glazier (2015) for comprehensive reviews, assessments and critiques of this field. We have chosen to work with this model since it attempts to quantify important ecological patterns and parameters by adopting a mechanistic perspective based on generic physical and chemical considerations, without the necessity for invoking complex (and specific) biological factors. Naturally, this approach has attracted a fair amount of criticism as outlined in the aforementioned references. Nevertheless, we will operate under the premise that the basic underpinnings of the MTE are valid for ecosystems on other planets, at least for those capable of sustaining life-as-we-know-it.

The MTE is founded on the principle that the metabolic rate, which serves as its cornerstone, scales as,

$$
B \propto m^{3 / 4} \exp \left(-\frac{E}{k_{\mathrm{B}} T}\right),
$$

where $m$ is the mass of the organism, $k_{\mathrm{B}}$ is the Boltzmann constant, $T$ is the absolute temperature, and $E$ is the average activation energy, which is determined by considering the appropriate rate-limiting step in metabolism (Gillooly et al. 2001). Now, suppose that one wished to formulate a 'mean' metabolic rate $\bar{B}$ across all species. This can be done by introducing the distribution function for the number of individuals with a given mass $N(m)$ as follows,

$$
\bar{B}=\frac{\int B(m, T) N(m) d m}{\int N(m) d m} \propto \exp \left(-\frac{E}{k_{\mathrm{B}} T}\right) .
$$

The last scaling follows if $E(m, T) \approx E(T)$, i.e. provided that the activation energy displays a weak dependence on the organism's mass. ${ }^{2}$ This appears to be a fairly robust assumption on Earth, since $E$ falls within a fairly narrow band of energies ranging between 0.6 and $0.7 \mathrm{eV}$, for unicellular organisms, plants, ectotherms and endotherms (Gillooly et al. 2001; Brown et al. 2004). It was pointed out in Gillooly et al. (2006) that the mean value of $E=0.65 \mathrm{eV}$ is nearly equal to the average activation energy of $0.66 \mathrm{eV}$ that arises from the rate of ATP synthesis in isolated mitochondria.

One of the central predictions of the MTE is that several ecological parameters are regulated by the metabolic rate, and are

\footnotetext{
${ }^{2}$ We have also assumed that the temperature dependence of the distribution function $N$ is minimal compared with its mass dependence.
}

thus expected to depend on the temperature via the Boltzmann factor inherent in equation (1). Examples of these parameters, which have been studied empirically, include:

- The production and turnover of biomass, and the rate of biological energy flux per unit area (Enquist et al. 2003; Brown et al. 2004).

- The maximal rates of population growth and molecular evolution (Brown et al. 2004; Savage et al. 2004; Gillooly et al. 2005), and the reciprocal developmental time (Gillooly et al. 2002).

- The rates of genetic divergence and speciation, species diversity and coexistence, and trophic interactions (Allen et al. 2002, 2006; Dell et al. 2014).

- Higher biodiversity is predicted to be prevalent in habitats with hotter average temperatures (Marquet et al. 2005; Allen et al. 2006; Fuhrman et al. 2008; Wang et al. 2009). This pattern has been proven to be valid on Earth, which is characterized by distinctive latitudinal gradients in species richness (Gaston 2000), although other factors, such as the degree of precipitation, also play an important (but possibly sub-dominant) role (Moles et al. 2014).

The Boltzmann factor dependence implies that all of the above quantities are expected to monotonically increase with temperature. This is closely related to the notion that 'Hotter is better', which posits that a higher value of $T$ is correlated with enhanced growth, fitness and diversity (Thompson 1942; Kingsolver \& Huey 2008; Mayhew et al. 2012), up to a particular limit.

At this stage, some important caveats are in order. The first stems from critiques concerning the validity and interpretation of the MTE (Clarke \& Fraser 2004; Clarke 2006; Downs et al. 2008; Price et al. 2012; Glazier 2015). In addition, the monotonically increasing trend with temperature cannot continue ad infinitum since thermal adaptation breaks down beyond a certain point (Angilletta 2009; Corkrey et al. 2012; Schulte 2015). Moreover, there exist several important and subtle ambiguities in resolving the exact relationship between temperature and the aforementioned traits (Clarke 2006; Clarke \& Rothery 2008; Kingsolver 2009). Lastly, our analysis has presupposed a steady-state temperature, but rapid fluctuations can engender mass extinctions and irreversible changes in the biosphere (Purvis \& Hector 2000; Barnosky et al. 2012; Frank \& Sullivan 2014).

\section{The temperature-dependent likelihood function}

Based on the preceding discussion, we introduce the likelihood factor for biodiversity which has been adapted from equation (2),

$$
\exp \left(-\frac{E}{k_{\mathrm{B}} T}\right) \theta\left(T-T_{\mathrm{L}}\right) \theta\left(T_{\mathrm{U}}-T\right),
$$

where $E$ and $T$ are now taken to be the mean activation energy and temperature respectively. The $\theta$ is the Heavyside function, which ensures that the likelihood becomes zero for $T<T_{\mathrm{L}}$ and $T>T_{\mathrm{U}}$.

The limits of Earth-based lifeforms range from 262 to $395 \mathrm{~K}$ (Rothschild \& Mancinelli 2001; McKay 2014), while the 
corresponding range for photosynthetic lifeforms is narrower since the upper bound is lowered to $348 \mathrm{~K}$ (Kiang et al. 2007). Note that $T$ corresponds to the average surface temperature of the planet in our subsequent analysis, because we are interested in quantifying the likelihood on a planetary scale. Thus, instead of $T$ representing the temperature of the local habitat (for ectotherms) or the average internal temperature (for endotherms), we have replaced it by the overall planetary surface temperature. Naturally, a limitation of this methodology is that it represents a coarse-grained estimate that smears out local effects. ${ }^{3}$

We are now in a position to construct the 'normalized' (Earth-referenced) temperature-based likelihood with respect to the Earth,

$$
\mathcal{P}_{\mathrm{T}}=\exp [-26.7(\delta-1)] \theta\left(T-T_{\mathrm{L}}\right) \theta\left(T_{\mathrm{U}}-T\right),
$$

where $T_{\mathrm{U}}$ and $T_{\mathrm{L}}$ should be interpreted as the limits over which the Boltzmann factor is valid. Clearly, the simple ansatz exemplified by equation (3) corresponds to sharp (discontinuous) cutoffs, and we refer the reader to Corkrey et al. (2012); Dell et al. (2014); Schulte (2015); Corkrey et al. (2016), wherein more sophisticated and realistic variants have been delineated. The temperature range $T_{\mathrm{L}}<T<T_{\mathrm{U}}$ is not expected to exceed the Earth-based photosynthesis limits for life-as-we-know-it. ${ }^{4} \mathrm{We}$ have also introduced the auxiliary parameter $\delta$ in equation (4),

$$
\delta=\frac{E}{E_{\oplus}} \frac{T_{\oplus}}{T} \sim \frac{T_{\oplus}}{T},
$$

where $E_{\oplus}=0.66 \mathrm{eV}$ (Dell et al. 2011) and $T_{\oplus}=287 \mathrm{~K}$ are the corresponding values for the Earth. The last relation follows from $E \sim E_{\oplus}$ since we only consider life-as-we-know-it in our present analysis. If we substitute $T=218 \mathrm{~K}$ for Mars, we find that $\mathcal{P}_{\mathrm{T}}=0$ because of the Heavyside function. In contrast, the equatorial temperatures on Mars can exceed $T_{\mathrm{L}}$, thereby giving rise to a finite value of $\mathcal{P}_{\mathrm{T}}$ locally. If we consider Venus instead, it is evident that $\mathcal{P}_{\mathrm{T}}=0$ since $T \gg T_{\mathrm{U}}$.

Thus, to conclude, we have hypothesized that equation (4) quantifies the likelihood of complex life-sustaining processes as a function of the planet's surface temperature. ${ }^{5}$ This function serves as a proxy for the (relative) metabolic rate, which, in turn, has been hypothesized to regulate important biological parameters, such as the species diversity, biological fluxes, and the rates of speciation and growth to name a few. Hence, if abiogenesis had been successfully initiated on a particular planet, the chances of complex life emerging are expected to be correspondingly greater for higher temperatures since: (i) the rates of evolution and speciation are enhanced, and (ii) a greater diversity of species are potentially sustainable. Hence, this

\footnotetext{
${ }^{3}$ Our approach is somewhat analogous to that of metapopulation ecology, wherein each 'unit' is a population patch comprising of several individuals; hence, a metapopulation is perceived as a 'population of populations' (Levins 1969).

${ }^{4}$ The assumption can be easily relaxed, and, indeed, a rich array of ecosystems based on alternative biochemistries have been extensively investigated (Bains 2004; Schulze-Makuch \& Irwin 2008).

${ }^{5}$ In Section 2.3 of Hoehler (2007), the temperature dependence of the metabolic rate was briefly discussed from the perspective of bioenergetics.
}

metric arguably constitutes a more sophisticated variant of understanding the likelihood of macroecological processes on exoplanets. However, we must reiterate that this likelihood function is not synonymous with a planet being habitable since there are myriad factors involved in the latter. Furthermore, equation (4) does not quantify the likelihood of abiogenesis, and the existence of the aforementioned ecological and evolutionary processes is obviously contingent upon life successfully originating (and diversifying) on the planet.

Although we have not directly estimated the prospects for abiogenesis on exoplanets, we wish to point out that several studies have presented empirical and theoretical evidence favouring a high-temperature origin of life (OOL; Pace 1991; Gaucher et al. 2003; Martin et al. 2008; Akanuma et al. 2013), although many factors still remain poorly known (Miller \& Lazcano 1995). If life did indeed originate in a hightemperature environment, perhaps the likelihood of abiogenesis could exhibit a Boltzmann factor dependence on the temperature akin to equation (2), thereby favouring thermophilic ancestral lifeforms (Weiss et al. 2016). However, an important point worth highlighting is that the temperature alluded to when discussing abiogenesis always represents the in situ value (e.g. at hydrothermal vents), and not the global planetary temperature.

\section{The role of atmospheric escape}

Next, we explore the constraints on the likelihood (of a planet being habitable) that are set by atmospheric escape. Before doing so, a few general observations are in order.

The phase diagram of water requires external pressure in order for liquid water to emerge upon warming solid ice (McKay 2014). It is therefore widely presumed that complex, surface-based, organic chemistry corresponding to life-as-weknow-it necessitates the existence of an atmosphere (Lammer et al. 2009; Cockell et al. 2016). However, atmospheres can be eroded through a diverse array of processes, such as thermal escape, photochemical escape and multiple non-thermal mechanisms such as sputtering (Johnson et al. 2008; Seager 2010; Lammer 2013). ${ }^{6}$ On the other hand, they can also be replenished through volcanism, giant impacts and evaporation of oceans (Kasting \& Catling 2003). If the associated timescales for the escape processes are 'fast', there may not exist sufficient time for complex life to emerge and evolve. Thus, we shall suppose henceforth that the timescales for atmospheric escape, which can be quantified in some instances, will serve as effective constraints for determining the inclination towards habitability of a given exoplanet relative to Earth.

In actuality, there are several important and distinct timescales that must be taken into account in conjunction with the planet's age $\left(t_{\mathrm{P}}\right)$. Some of the notable ones are as follows:

\footnotetext{
${ }^{6}$ In addition, planets could lose a significant fraction of their atmospheres due to X-ray and ultraviolet irradiation from supermassive black holes (Forbes \& Loeb 2017).
} 
- The characteristic timescale(s) involved in the depletion of the planetary atmosphere $\left(t_{\ell}\right)$, which will be the focus of this paper.

- The minimum timescale required for abiogenesis - the $\mathrm{OOL}$ - to commence $\left(t_{\mathrm{OOL}}\right)$, since the likelihood of life arising on the planet is zero for $t_{\mathrm{P}}<t_{\mathrm{OOL}}$. As per current evidence, it seems plausible that $t_{\mathrm{OOL}} \leqslant 500 \mathrm{Myr}$ on Earth (Bell et al. 2015; Dodd et al. 2017) although, in light of the many uncertainties involved, this timescale ought not be perceived as being definitive.

- The timescale over which the planet will be subjected to the extended pre-main-sequence (pre-MS) phase $\left(t_{\mathrm{PMS}}\right)$, since this can adversely impact habitability (extreme water loss), especially when dealing with exoplanets in the $\mathrm{HZ}$ of M-dwarfs (Ramirez \& Kaltenegger 2014; Luger \& Barnes 2015; Tian \& Ida 2015).

- The duration of time taken for a given planet to 'enter' the outer edge of the $\mathrm{HZ}\left(t_{\mathrm{OHZ}}\right)$, and to finally 'exit' the inner edge of the $\mathrm{HZ}\left(t_{\mathrm{IHZ}}\right)$, since the $\mathrm{HZ}$ itself evolves over time (Rushby et al. 2013). By construction, $t_{\mathrm{P}}<t_{\mathrm{OHZ}}$ or $t_{\mathrm{P}}>t_{\mathrm{IHZ}}$ imply the planet would not be habitable (in the conventional sense).

Thus, we shall implicitly restrict our attention to planets where $t_{\mathrm{P}}>t_{\mathrm{OOL}}, \mathrm{t}_{\mathrm{OHZ}}<t_{\mathrm{P}}<t_{\mathrm{IHZ}}$, and the pre-MS phase has not rendered the planet uninhabitable.

\section{Timescales for atmospheric escape}

As noted earlier, there are several mechanisms that lead to atmospheric losses. In our analysis, we shall consider two dominant causes, namely, hydrodynamic escape and stellar wind stripping. We do not evaluate the extent of Jeans escape, as it does not play a major role in facilitating escape of heavier molecules (such as $\mathrm{O}_{2}$ and $\mathrm{CO}_{2}$ ) from Earth-like planets in the $\mathrm{HZ}$ (Lammer 2013).

For hydrodynamic escape, we rely upon the assumption of energy-limited escape (Seager 2010), enabling us to estimate the lifetime of the planet's atmosphere as,

$$
t_{\mathrm{HD}} \sim \frac{G M_{\mathrm{p}}}{\pi R_{\mathrm{p}}^{3}} \frac{M_{\text {atm }}}{\beta \eta\left\langle F_{\mathrm{EUV}}\right\rangle},
$$

where $M_{\mathrm{atm}}, M_{\mathrm{p}}$ and $R_{\mathrm{p}}$ are the mass of the atmosphere, mass and radius of the planet, respectively; $\left\langle F_{\mathrm{EUV}}\right\rangle$ is the average extreme ultraviolet (EUV) flux while $\beta$ and $\eta$ are phenomenological parameters. Further details concerning the derivation of equation (6) can be found in Chapter 4 of Seager (2010) and Section 2 of Owen \& Alvarez (2016). Other forms of UV-driven atmospheric loss include recombination-limited and photon-limited escape (Owen \& Alvarez 2016).

Next, we consider stripping of the atmosphere by the stellar wind. The associated timescales are given by $t_{\mathrm{MSW}}$ and $t_{\mathrm{UMSW}}$ for the magnetized and unmagnetized cases (with and without an intrinsic dipole field). Note that we are interested in the fastest timescale for atmospheric loss, thereby leading us to $t_{\ell}=\min \left\{t_{\mathrm{HD}}, t_{\mathrm{UMSW}}\right\}$ or $t_{\ell}=\min \left\{t_{\mathrm{HD}}, t_{\mathrm{MSW}}\right\}$ depending on the situation. However, the escape rates for magnetized planets are usually expected to be somewhat lower (Lammer 2013;
Ehlmann et al. 2016; Dong et al. 2017b), implying that $t_{\mathrm{UMSW}}<t_{\mathrm{MSW}}$.

In fact, for most exoplanets in the $\mathrm{HZ}$ around $M$-dwarfs, the dynamic pressure exerted by the stellar wind is so great that the additional shielding offered by the planet's magnetic field $B_{\mathrm{P}}$ is relatively unimportant (Garraffo et al. 2016; Airapetian et al. 2017; Dong et al. 2017b), provided that $B_{\mathrm{P}}$ is not anomalously high (Vidotto et al. 2013). By utilizing standard dynamo scaling laws (Christensen 2010), it can be shown that $B_{\mathrm{P}}$ will not be very large if the convected heat flux in the planetary core is not significantly higher than that of the Earth. In fact, tidally locked exoplanets, expected to be fairly common in the HZ around $M$-dwarfs, are typically associated with weak magnetic moments (Khodachenko et al. 2007; Shields et al. 2016).

Based on the above set of arguments, we may henceforth adopt $t_{\mathrm{UMSW}} \sim t_{\mathrm{MSW}}$ for certain $M$-dwarf exoplanets. The timescale of atmospheric loss for unmagnetized planets $\left(t_{\mathrm{SW}}\right)$ is given by,

$$
t_{\mathrm{SW}} \sim \frac{2}{\alpha} \frac{M_{\mathrm{atm}}}{\dot{M}_{\star}}\left(\frac{a}{R_{\mathrm{P}}}\right)^{2}
$$

where $a$ is the semi-major axis of the planet, $\dot{M}_{\star}$ is the stellar mass loss rate and $\alpha$ is the entrainment efficiency that is treated as a constant (Zendejas et al. 2010). The above formula was verified to be fairly accurate by means of numerical simulations in Dong et al. (2017a).

\section{Contribution of atmospheric loss to the likelihood function}

Following our preceding discussion, the atmospheric loss is given by $t_{\ell}=\min \left\{t_{\mathrm{HD}}, t_{\mathrm{SW}}\right\}$, where $t_{\mathrm{HD}}$ and $t_{\mathrm{Sw}}$ are given by equations (6) and (7), respectively. We define the normalized (Earth-referenced) likelihood of this timescale in the following manner:

$$
\mathcal{P}_{\mathrm{A}}=\frac{t_{\ell}}{t_{\oplus}},
$$

where $t_{\oplus}$ is the corresponding value of $t_{\ell}$ upon substituting Earth's parameters. When $t_{\mathrm{HD}}<t_{\mathrm{SW}}$, we find that equation (8) simplifies to,

$$
\mathcal{P}_{\mathrm{A}}(\mathrm{HD})=\left(\frac{P_{\mathrm{s}}}{1 \mathrm{~atm}}\right)\left(\frac{R_{\mathrm{P}}}{R_{\oplus}}\right)\left(\frac{\left\langle F_{\mathrm{EUV}}\right\rangle}{\left\langle F_{\oplus}\right\rangle}\right)^{-1},
$$

where $P_{\mathrm{s}}=g M_{\text {atm }} /\left(4 \pi R_{\mathrm{p}}^{2}\right)$ is the surface pressure of the atmosphere, $F_{\oplus}$ is the value of $\left\langle F_{\mathrm{EUV}}\right\rangle$ for the Earth and we have employed the mass-radius relation $M / M_{\oplus} \sim\left(R / R_{\oplus}\right)^{3.7}$ (Valencia et al. 2006; Zeng et al. 2016). Similarly, when $t_{\mathrm{HD}}>t_{\mathrm{SW}}$, it is easy to verify that equation (8) can be expressed as,

$$
\mathcal{P}_{\mathrm{A}}(\mathrm{SW})=\left(\frac{P_{\mathrm{s}}}{1 \mathrm{~atm}}\right)\left(\frac{a}{1 \mathrm{AU}}\right)^{2}\left(\frac{R_{\mathrm{P}}}{R_{\oplus}}\right)^{-1.7}\left(\frac{\dot{M}_{\star}}{\dot{M}_{\odot}}\right)^{-1},
$$

where $\dot{M}_{\odot}$ is the Sun's mass-loss rate. In general, the mass-loss rate displays a complex empirical dependence on stellar parameters, for instance the star's mass, activity, age and rotation rate (Cranmer \& Saar 2011). We shall adopt the scaling relation proposed in Johnstone et al. (2015) for low-mass stars (although its validity remains somewhat uncertain for 
$M_{\star}<0.4 M_{\odot}$ and $\left.M_{\star}>1.1 M_{\odot}\right):$

$$
\frac{\dot{M}_{\star}}{\dot{M}_{\odot}}=\left(\frac{R_{\star}}{R_{\odot}}\right)^{2}\left(\frac{\Omega_{\star}}{\Omega_{\odot}}\right)^{1.33}\left(\frac{M_{\star}}{M_{\odot}}\right)^{-3.36}
$$

where $R_{\star}, \Omega_{\star}$ and $M_{\star}$ are the stellar radius, rotation rate and mass, respectively. When we substitute the values for Proxima Centauri, we find that $\dot{M}_{\star} \approx 4.8 \dot{M}_{\odot}$, which is fairly close to $\dot{M}_{\star} \approx \dot{M}_{\odot}$ obtained from simulations (Garraffo et al. 2016). The above expression is based on the assumption that the star does not rotate rapidly, ${ }^{7}$ since the mass-loss rate would otherwise attain a saturation value with the dependence $\dot{M}_{\star} \propto M_{\star}^{1.3}$ in that regime (Johnstone et al. 2015).

A few general observations can be drawn from equations (9) and (10). The likelihood function is linearly proportional to the surface pressure (which itself is related to $M_{\mathrm{atm}}$ ), implying that planets with relatively massive atmospheres are conducive to being superhabitable, in agreement with Vladilo et al. (2013) and Heller \& Armstrong (2014). Secondly, we observe that exoplanets in the $\mathrm{HZ}$ around $M$-dwarfs are typically subject to higher values of $\left\langle F_{\mathrm{EUV}}\right\rangle$ compared with the Earth (France et al. 2013), and are also located much closer (Kopparapu et al. 2013). When combined with equations (9) and (10), these facts imply that $\mathcal{P}_{\mathrm{A}}$ for such exoplanets is likely to be much lower than unity.

We have seen that $t_{\ell}$ quantifies the timescale over which the atmosphere is present. Over this duration, it is worth highlighting that species diversity itself increases over time. The enhancement of biodiversity has been predicted to obey logistic growth (Purvis \& Hector 2000; Benton 2009), which can, in some instances, be loosely visualized as a linear function during the growing phase prior to saturation. Thus, one could, perhaps, also envision equation (8) as a heuristic measure of the maximal species diversity relative to Earth. In turn, a planetary ecosystem with higher biodiversity would be typically associated with greater stability and multifunctionality (Hooper et al. 2005; Cardinale et al. 2012), although the subtleties inherent in analyses of diversity-stability relationships should be duly recognized (Ives \& Carpenter 2007).

Lastly, we observe that $\mathcal{P}_{\mathrm{A}}=0$ when $t_{\ell}<t_{\mathrm{OOL}}$ since the planet's atmosphere is lost prior to the onset of abiogenesis. Similarly, if $t_{\ell}>t_{\mathrm{HZ}}:=t_{\mathrm{IHZ}}-t_{\mathrm{OHZ}}$, one must replace $t_{\ell}$ in equation (8) with $t_{\mathrm{HZ}}$ since the latter would become the critical timescale in this regime.

\section{The likelihood function and its consequences}

We are now in a position to define the overall likelihood function $\mathcal{P}$ from the preceding results,

$$
\mathcal{P}=\mathcal{P}_{\mathrm{T}} \cdot \mathcal{P}_{\mathrm{A}},
$$

where $\mathcal{P}_{\mathrm{T}}$ is given by equation (4) and $\mathcal{P}_{\mathrm{A}}$ corresponds to either equation (9) or equation (10) depending on the dominant process that drives atmospheric escape.

\footnotetext{
${ }^{7}$ A rapid rotation rate corresponds to a value that is close to the saturation estimate of $\Omega_{\text {sat }}=15 \Omega_{\odot}\left(M_{\star} / M_{\odot}\right)^{2.3}$ (Johnstone et al. 2015).
}

This function can be used to determine the likelihood of a planet being conducive to life relative to Earth. The chief advantage of our methodology is that nearly all of the parameters are direct observables, or can be deduced indirectly, by means of numerical simulations. The two primary uncertainties involve the surface pressure $P_{\mathrm{s}}$ and the surface temperature $T$. In our subsequent analysis, we shall suppose that the surface pressure is equal to that of the Earth. This still leaves us with $\delta$, which is defined in equation (5). We introduce the ansatz $T=\zeta T_{\text {eq }}$, where $T_{\text {eq }}$ is the equilibrium temperature and $\zeta$ is a phenomenological parameter that captures the effects of greenhouse warming, snowball dynamics, tidal heating and other feedback mechanisms. In general, $\zeta$ is not constant across all planets, but we adopt this simplifying assumption in order to derive the likelihood function for some of the recently discovered exoplanets in Table 1.

We find that the likelihood function $\mathcal{P}_{\mathrm{A}}$ for Proxima $b$ and TRAPPIST-1e is about 1-2 orders of magnitude lower than that of Earth if the atmospheric escape is dominated by hydrodynamic escape. If the atmospheric losses occur due to stellar wind erosion, we conclude that the likelihood is even lower (by three orders of magnitude) for these planets. Another interesting result is that TRAPPIST-1e exhibits a higher likelihood of being habitable (albeit by only a factor of a few) when compared with Proxima $b$. When dealing with Proxima $b$, the ostensibly habitable planets of the TRAPPIST-1 system, and other exoplanets in the HZ of $M$-dwarfs, it is equally important to realize that the timescales for atmospheric loss may be sufficiently short such that $t_{\ell}<t_{\mathrm{OOL}}$, thereby implying that abiogenesis will be non-functional on these planets.

When we consider TRAPPIST- $1 f$ and TRAPPIST- $1 g$, the overall likelihood function ends up being zero in our simplified model because $\mathcal{P}_{\mathrm{T}}=0$ for $T<T_{\mathrm{L}}$. We reiterate that this only represents a coarse-grained, global likelihood; in reality, there may exist locally favourable temperatures, perhaps near the terminator line, for these two planets enabling $\mathcal{P}_{\mathrm{T}} \neq 0$ to occur in these regions. Our conclusions pertaining to these two planets are also broadly consistent with the results obtained from three-dimensional climate simulations (Wolf 2017), although the latter study (as well as our model) does not include the effects of tidal heating that are likely to be significant in the TRAPPIST-1 exoplanetary system.

In Fig. 1, we plot $\mathcal{P}_{\mathrm{T}}$, given by equation (4), as a function of the planet's surface temperature $T$. This plot can be interpreted as the likelihood of the Earth sustaining a complex biosphere provided that it was characterized by a steady surface temperature different from its current value. The presence of the exponential function ensures that the likelihood can vary over two orders of magnitude for a relatively narrow range of $T$. This serves to underscore the fact that a variety of macroecological processes are quite sensitive to the temperature, implying that the latter parameter will clearly play a central role in discussions of planetary habitability.

Upon inspecting equation (9) next, we find that it depends on both planetary and stellar parameters. We shall focus on planets that are 'Earth-like' (but only in a superficial sense), i.e. the surface pressure and radius are chosen to equal the 
Table 1. The likelihood function relative to Earth for different $H Z$ exoplanets

\begin{tabular}{|c|c|c|c|c|c|}
\hline Planet & $\mathcal{P}_{\mathrm{T}}$ & $\mathcal{P}_{\mathrm{A}}(\mathrm{HD})$ & $\mathcal{P}_{\mathrm{A}}(\mathrm{SW})$ & $\mathcal{P}(\mathrm{HD})$ & $\mathcal{P}(\mathrm{SW})$ \\
\hline Earth & 1 & 1 & 1 & 1 & 1 \\
\hline Proxima $b$ & 0.13 & $3 \times 10^{-2}$ & $2 \times 10^{-3}$ & $3.9 \times 10^{-3}$ & $2.6 \times 10^{-4}$ \\
\hline TRAPPIST-1e & 0.93 & $4.5 \times 10^{-2}$ & $9.1 \times 10^{-4}$ & $4.2 \times 10^{-2}$ & $8.5 \times 10^{-4}$ \\
\hline TRAPPIST- $1 f$ & 0 & $8.7 \times 10^{-2}$ & $1.3 \times 10^{-3}$ & 0 & 0 \\
\hline TRAPPIST- $1 g$ & 0 & 0.14 & $1.7 \times 10^{-3}$ & 0 & 0 \\
\hline
\end{tabular}

$\mathcal{P}_{\mathrm{A}}(\mathrm{HD})$ and $\mathcal{P}_{\mathrm{A}}(\mathrm{SW})$ are given by equations (9) and (10), respectively, while $\mathcal{P}(\mathrm{HD})=\mathcal{P}_{\mathrm{T}} \cdot \mathcal{P}_{\mathrm{A}}(\mathrm{HD})$ and $\mathcal{P}(\mathrm{SW})=\mathcal{P}_{\mathrm{T}} \cdot \mathcal{P}_{\mathrm{A}}(\mathrm{SW})$. The stellar mass-loss rate for Proxima Centauri is from Garraffo et al. (2016) (see also Wood et al. 2001) and the corresponding value for TRAPPIST-1 has been assumed to be approximately equal to that of Proxima Centauri. The stellar and planetary parameters have been tabulated in Anglada-Escudé et al. (2016) and Gillon et al. (2017), while EUV fluxes were taken from Ribas et al. (2016) and Bourrier et al. (2017) (see also Bolmont et al. 2017) for Proxima $b$ and the TRAPPIST-1 system, respectively.

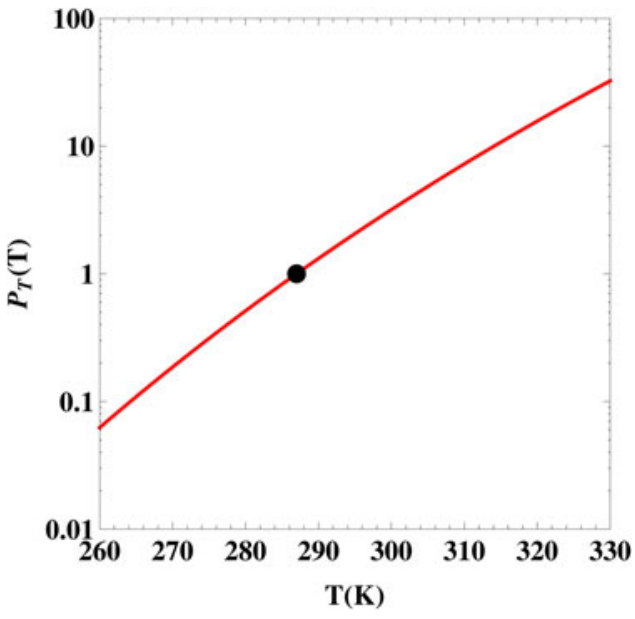

Fig. 1. The likelihood as a function of the planet's average surface temperature $T$. The black dot represents the Earth's value at $T=287 \mathrm{~K}$.

Earth's values. Figure 2 depicts the plot of $\mathcal{P}_{\mathrm{A}}(\mathrm{HD})$ as a function of $\left\langle F_{\text {EUV }}\right\rangle$. Physically speaking, this figure quantifies the likelihood function of energy-limited atmospheric escape if the Earth were subjected to varying degrees of EUV flux.

Finally, we turn our attention to equation (10). This equation involves a large number of planetary and stellar parameters as well. As before, we consider an 'Earth clone' with physical parameters equal to that of Earth, namely, $P_{\mathrm{s}} \sim 1$ atm, $R_{\mathrm{P}} \sim R_{\oplus}$ and $T_{\text {eq }} \sim T_{\text {eq, } \oplus \text {. If we further use } L_{\star} \propto M_{\star}^{3}}^{3}$ (mass-luminosity relationship), $R_{\star} \propto M_{\star}^{0.8}$ (Johnstone et al. 2015) and $a \propto L_{\star}^{1 / 2}$ for a fixed value of $T_{\text {eq }}$ and the Bond albedo, we end up with the scalings,

$$
\mathcal{P}_{\mathrm{A}}(\mathrm{SW}) \approx\left(\frac{\Omega_{\star}}{\Omega_{\odot}}\right)^{-1.33}\left(\frac{M_{\star}}{M_{\odot}}\right)^{4.76}
$$

for the likelihood of the Earth's atmosphere to persist which is a necessary although not sufficient condition for habitability - in the HZ of a different star. Thus, we can immediately see that a slowly rotating, higher mass star is more conducive to hosting life on an Earth clone. Note that the above formula is not valid for rapidly rotating stars that yield scaling relations different from the Sun; a similar analysis using the corresponding formula for $\dot{M} \star$ from Johnstone $e t$ al.

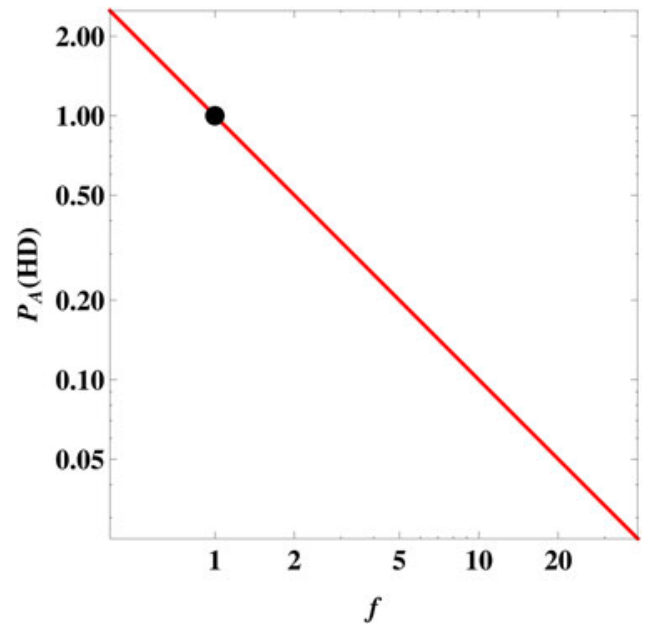

Fig. 2. The likelihood as a function of the normalized EUV flux $f=\left\langle F_{\mathrm{EUV}}\right\rangle /\left\langle F_{\oplus}\right\rangle$ for an Earth clone. The black dot represents the Earth's position at $f=1$.

(2015) leads to,

$$
\mathcal{P}_{\mathrm{A}}(\mathrm{SW}) \approx 2.7 \times 10^{-2}\left(\frac{M_{\star}}{M_{\odot}}\right)^{1.7},
$$

which does not exhibit an $\Omega_{\star}$ dependence. This expression also implies that stars with a higher mass are more likely to host planets in the $\mathrm{HZ}$ that are capable of possessing long-term atmospheres. ${ }^{8}$ We reiterate that equations (13) and (14) are only valid for an Earth clone, and, in reality, $\mathcal{P}_{\mathrm{A}}(\mathrm{SW})$ depends both on stellar and planetary parameters as seen from equation (10). We have plotted the results from equations (13) and (14) in the two panels of Fig. 3.

\section{Conclusions}

In this paper, we have attempted to address the important question of habitability metrics from a more quantitative

${ }^{8}$ The overall lifetime of a star scales with its mass as $M_{\star}^{-p}$, where $2<p<3$ (Loeb et al. 2016). For a sufficiently massive star, its lifetime will be lower than $t_{O O L}$, implying that the likelihood of life would become zero. Hence, the predicted increase of $\mathcal{P}_{A}(\mathrm{SW})$ with stellar mass is valid only up to a cutoff value. 

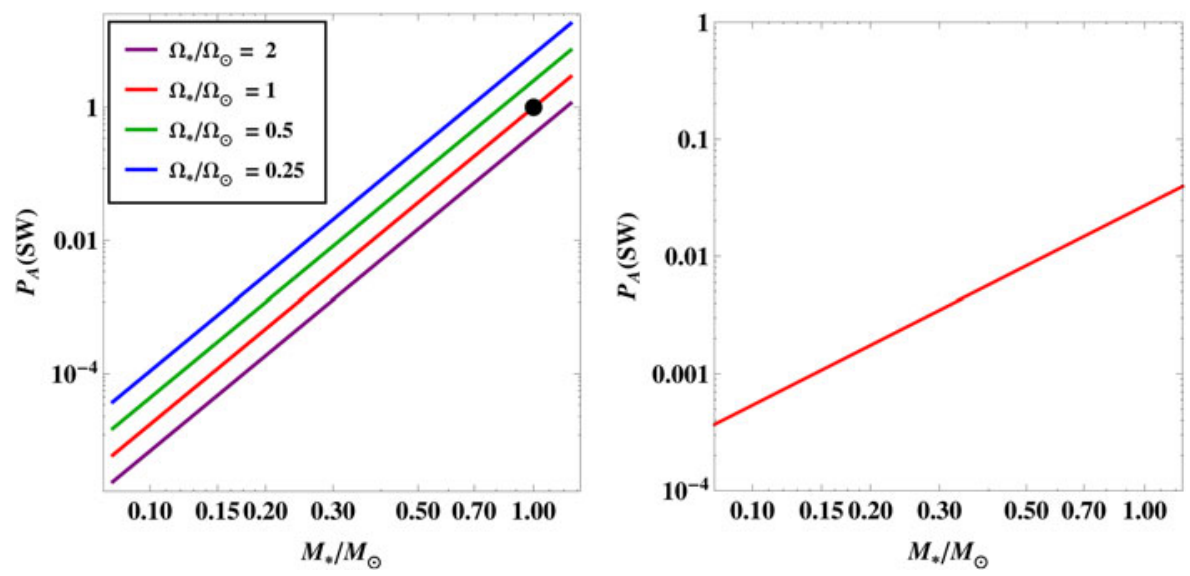

Fig. 3. The likelihood as a function of the stellar mass for an Earth clone. In the left-hand panel, each plot corresponds to a different value of the rotation rate $\Omega_{\star}$. The black dot signifies the position of the Sun. In the right-hand panel, the likelihood has been plotted for rapidly rotating stars, in which case it does not depend on $\Omega_{\star}$.

perspective. We begin with the caveat that our work entails a certain degree of terracentrism with an emphasis on life-as-weknow-it; the associated assumptions cannot be easily bypassed since the Earth is the only planet that is presently known to harbour life. In addition, a genuinely quantitative understanding of habitability is not feasible at this stage given that there exist far too many unknowns (Schulze-Makuch \& Guinan 2016; Tasker et al. 2017).

We have attempted to: (i) draw upon models with strong physical underpinnings, and (ii) present the results in terms of basic physical parameters that can be determined via observations or simulations. We focused on two major physical processes, namely the role of planetary temperature and atmospheric escape. The former was analysed by means of the MTE, which predicts that many macroecological processes exhibit a Boltzmann factor dependence on the temperature within a certain range. We addressed the possibility of atmospheric escape by considering two different mechanisms, namely hydrodynamic escape and stellar wind-induced stripping.

Our analysis gave rise to a diverse array of conclusions, which have been summarized below:

- Planets with a warmer mean surface temperature are more conducive to being habitable, insofar macroscopic ecological (as well as evolutionary) processes are concerned, albeit only up to a limited temperature range.

- Planets with higher surface pressure are, ceteris paribus, more likely to be habitable. ${ }^{9}$ It has also been argued by some authors that abiogenesis on Earth was initiated in a high-pressure environment (Martin et al. 2008; Picard \& Daniel 2013), consequently indicating that pressure may play a potentially positive role in the OOL.

\footnotetext{
9 This statement is manifestly valid only in the absence of additional negative consequences arising from a massive atmosphere, such as a runaway greenhouse effect (e.g. Venus), which would otherwise make the planet uninhabitable.
}

- For an 'Earth clone' around a different star, the likelihood of retaining its atmosphere increases with the star's mass, while decreasing with rotation rate and the average ultraviolet (EUV) flux. High doses of ionizing radiation are also likely to have deleterious effects on the functioning of organic molecules and organisms (Dartnell 2011), although the beneficial effects of UV radiation in the context of prebiotic chemistry have been well documented (Ranjan \& Sasselov 2016).

- On account of the above reasons, Earth-sized planets in the $\mathrm{HZ}$ around $M$-dwarfs are presumably much less likely to be habitable, conceivably by several orders of magnitude, when compared with the Earth-Sun system. Hence, even though $M$-dwarfs outnumber other stars in our Galaxy, we propose that future searches for life on exo-Earths (Horner \& Jones 2010) should prioritize a subset of $G$ - and $K$-type stars (Tian \& Ida 2015; Cuntz \& Guinan 2016). This may also explain why we live on a terrestrial planet currently in the $\mathrm{HZ}$ of the Sun, and not one that is in the $\mathrm{HZ}$ of an $M$-dwarf in the cosmic future (Loeb et al. 2016; HaqqMisra et al. 2017).

- More specifically, Proxima $b$ and TRAPPIST-1e yield a much lower likelihood of being habitable (by 2-4 orders of magnitude), with respect to atmospheric escape mechanisms, when compared with Earth. Furthermore, TRAPPIST-1e appears to be more conducive to hosting life, while only by a factor of a few, with respect to Proxima $b$ if one makes the further assumption that their host stars have a similar stellar mass-loss rate.

- If the assumptions in this paper are valid, it seems plausible that TRAPPIST- $1 f$ and TRAPPIST- $1 g$ are incapable of sustaining life across a significant fraction of the surface, although the possibility of local life-bearing zones and life seeded by means of panspermia (Lingam 2016; Lingam \& Loeb 2017) cannot and ought not be ruled out.

- As most of the stellar and planetary parameters are timedependent, the likelihood functions are also implicitly 
dynamical. ${ }^{10}$ This fact is fully consistent with the notion that planetary habitability (and sustainability) evolves over time (Frank \& Sullivan 2014).

- Many of these findings, after suitable reinterpretation, are also applicable to habitable exomoons, which may outnumber habitable exoplanets (Heller et al. 2014).

We end by cautioning that our proposed methodology is by no means complete, since important (first-order) feedback mechanisms - such as the complex, non-linear and adaptive interplay between life and planetary habitability (Levins 1968; Lovelock \& Margulis 1974; Levin 1998; Angeli et al. 2004; Chopra \& Lineweaver 2016) - have not been included herein (Lineweaver \& Chopra 2012; Ehlmann et al. 2016; Judson 2017). We also wish to point out that we have altogether neglected second-order effects in this study. For instance, it has been suggested that a larger surface area facilitates higher biodiversity (Rosenzweig 1995), thereby making the planet superhabitable (Heller \& Armstrong 2014). However, it is important to recognize that the radius (and hence the area) of super-Earths is quite constrained (Rogers 2015; Chen \& Kipping 2017), thereby implying that this effect qualifies as an $\mathcal{O}(1)$ contribution.

As our work is founded on physical and mechanistic considerations inclined towards universality, we advocate the adoption of such an approach in future studies that seek to quantify the likelihood of exoplanets hosting complex and long-lived biospheres. Pursuing such lines of enquiry may also prove to be a natural and timely means of investigating the dependence of abiogenesis, at least for life-as-we-know-it, on biochemical (possibly even planetary and stellar) parameters by means of associated paradigms (Goldenfeld \& Woese 2011; Pascal et al. 2013; Davies \& Walker 2016), thereby complementing previous probabilistic estimates (Lineweaver \& Davis 2002; Carter 2008; Spiegel \& Turner 2012; Scharf \& Cronin 2016). Studies along these lines would lead us towards a resolution of the fundamental question as to whether life (and intelligence) in the universe is an extremely rare phenomenon (Simpson 1964; Monod 1971; Ward \& Brownlee 2000; Morris 2003) or an inevitable 'cosmic imperative' (de Duve 1995, 2011). ${ }^{11}$

\section{Acknowledgements}

The authors thank Chuanfei Dong, John Forbes, Jeffrey Linsky, Sukrit Ranjan, John Raymond, Ed Turner and the referee for their helpful comments concerning the paper. This work was supported in part by a grant from the Breakthrough Prize Foundation for the Starshot Initiative.

\footnotetext{
${ }^{10}$ For instance, the stellar mass-loss rate in equation (7) changes significantly, by several orders of magnitude, over time for Solar-like stars (Wood et al. 2002).

${ }^{11}$ With regards to the latter viewpoint, the reader may also consult the likes of Kauffman (1995); Morowitz \& Smith (2007); Martinez (2014); Bains \& Schulze-Makuch (2016) that explore similar perspectives.
}

\section{References}

Agutter, P.S. \& Wheatley, D.N. (2004). Metabolic scaling: consensus or controversy? Theor. Biol. Med. Model. 1(1), 13.

Airapetian, V.S., Glocer, A., Khazanov, G.V., Loyd, R.O.P., France, K., Sojka, J., Danchi, W.C. \& Liemohn, M.W. (2017). How hospitable are space weather affected habitable zones? The role of ion escape. Astrophys. J. Lett. 836, L3.

Akanuma, S., Nakajima, Y., Yokobori, S., Kimura, M., Nemoto, N., Mase, T., Miyazono, K., Tanokura, M. \& Yamagishi, A. (2013). Experimental evidence for the thermophilicity of ancestral life. Proc. Natl. Acad. Sci. USA 110(27), 11067-11072.

Allen, A.P., Brown, J.H. \& Gillooly, J.F. (2002). Global biodiversity, biochemical kinetics, and the energetic-equivalence rule. Science 297 (5586), 1545-1548.

Allen, A.P., Gillooly, J.F., Savage, V.M. \& Brown, J.H. (2006). Kinetic effects of temperature on rates of genetic divergence and speciation. Proc. Natl. Acad. Sci. USA 103(24), 9130-9135.

Angeli, D., Ferrell, J.E. Jr. \& Sontag, E.D. (2004). Detection of multistability, bifurcations, and hysteresis in a large class of biological positive-feedback systems. Proc. Natl. Acad. Sci. USA 101(7), 1822-1827.

Angilletta, M.J. (2009). Thermal Adaptation: A Theoretical and Empirical Synthesis. Oxford University Press, Oxford.

Anglada-Escudé, G. et al. (2016). A terrestrial planet candidate in a temperate orbit around Proxima Centauri. Nature 536(7617), 437-440.

Bains, W. (2004). Many chemistries could be used to build living systems. Astrobiology 4(2), 137-167.

Bains, W. \& Schulze-Makuch, D. (2016). The cosmic zoo: the (near) inevitability of the evolution of complex, macroscopic life. Life 6(3), 25.

Ball, P. (2008). Water as an active constituent in cell biology. Chem. Rev. 108 (1), 74-108.

Barnosky, A.D. et al. (2012). Approaching a state shift in Earth's biosphere. Nature 486(7401), 52-58.

Bell, E.A., Boehnke, P., Harrison, T.M. \& Mao, W.L. (2015). Potentially biogenic carbon preserved in a 4.1 billion-year-old zircon. Proc. Natl. Acad. Sci. USA 112(47), 14518-14521.

Benner, S.A., Ricardo, A. \& Carrigan, M.A. (2004). Is there a common chemical model for life in the universe? Curr. Opin. Chem. Biol. 8(6), 672-689.

Benton, M.J. (2009). The red queen and the court jester: species diversity and the role of biotic and abiotic factors through time. Science $\mathbf{3 2 3}$ (5915), 728

Bolmont, E., Selsis, F., Owen, J.E., Ribas, I., Raymond, S.N., Leconte, J. \& Gillon, M. (2017). Water loss from terrestrial planets orbiting ultracool dwarfs: implications for the planets of TRAPPIST-1. Mon. Not. R. Astron. Soc. 464(3), 3728-3741.

Bourrier, V., Ehrenreich, D., Wheatley, P.J., Bolmont, E., Gillon, M., de Wit, J., Burgasser, A.J., Jehin, E., Queloz, D. \& Triaud, A.H.M.J. (2017). Reconnaissance of the TRAPPIST-1 exoplanet system in the Lyman- $\alpha$ line. Astron. Astrophys. 599, L3.

Brown, J.H., Gillooly, J.F., Allen, A.P., Savage, V.M. \& West, G.B. (2004). Toward a metabolic theory of ecology. Ecology 85(7), 1771-1789.

Cardinale, B.J. et al. (2012). Biodiversity loss and its impact on humanity. Nature 486(7401), 59-67.

Carter, B. (2008). Five- or six-step scenario for evolution? Int. J. Astrobiol. 7 (2), 177-182.

Chen, J. \& Kipping, D. (2017). Probabilistic forecasting of the masses and radii of other worlds. Astrophys. J. 834(1), 17.

Chopra, A. \& Lineweaver, C.H. (2016). The case for a Gaian bottleneck: the biology of habitability. Astrobiology 16(1), 7-22.

Christensen, U.R. (2010). Dynamo scaling laws and applications to the planets. Space Sci. Rev. 152(1), 565-590.

Chyba, C.F. \& Hand, K.P. (2005). Astrobiology: the study of the living universe. Annu. Rev. Astron. Astrophys. 43, 31-74.

Clarke, A. (2006). Temperature and the metabolic theory of ecology. Funct. Ecol. 20(2), 405-412.

Clarke, A. \& Fraser, K.P.P. (2004). Why does metabolism scale with temperature? Funct. Ecol. 18(2), 243-251. 
Clarke, A. \& Rothery, P. (2008). Scaling of body temperature in mammals and birds. Funct. Ecol. 22(1), 58-67.

Cockell, C.S. et al. (2016). Habitability: a review. Astrobiology 16(1), 89-117.

Corkrey, R., Olley, J., Ratkowsky, D., McMeekin, T. \& Ross, T. (2012). Universality of thermodynamic constants governing biological growth rates. PLoS ONE 7(2), e32003.

Corkrey, R., McMeekin, T.A., Bowman, J.P., Ratkowsky, D.A., Olley, J. \& Ross, T. (2016). The biokinetic spectrum for temperature. PLOS ONE 11(4), e0153343.

Cossins, A.R. \& Bowler, K. (1987). Temperature Biology of Animals. Chapman \& Hall, London.

Cranmer, S.R. \& Saar, S.H. (2011). Testing a predictive theoretical model for the mass loss rates of cool stars. Astrophys. J. 741(1), 54.

Cuntz, M. \& Guinan, E.F. (2016). About exobiology: the case for dwarf K stars. Astrophys. J. 827(1), 79.

Dartnell, L.R. (2011). Ionizing radiation and life. Astrobiology 11(6), 551-582.

Davies, P.C.W. \& Walker, S.I. (2016). The hidden simplicity of biology. Rep. Prog. Phys. 79(10), 102601.

de Duve, C. (1995). Vital Dust: Life as a Cosmic Imperative. Basic Books, New York.

de Duve, C. (2011). Life as a cosmic imperative? Phil. Trans. R. Soc. A. 369 (1936), 620-623.

Dell, A.I., Pawar, S. \& Savage, V.M. (2011). Systematic variation in the temperature dependence of physiological and ecological traits. Proc. Natl. Acad. Sci. USA 108(26), 10591-10596.

Dell, A.I., Pawar, S. \& Savage, V.M. (2014). Temperature dependence of trophic interactions are driven by asymmetry of species responses and foraging strategy. J. Anim. Ecol. 83(1), 70-84.

Deutsch, C.A., Tewksbury, J.J., Huey, R.B., Sheldon, K.S., Ghalambor, C.K., Haak, D.C. \& Martin, P.R. (2008). Impacts of climate warming on terrestrial ectotherms across latitude. Proc. Natl. Acad. Sci. USA 105(18), 6668-6672.

Dillon, M.E., Wang, G. \& Huey, R.B. (2010). Global metabolic impacts of recent climate warming. Nature $\mathbf{4 6 7 ( 7 3 1 6 ) , 7 0 4 - 7 0 6 .}$

Dittmann, J.A. et al. (2017). A temperate rocky super-Earth transiting a nearby cool star. Nature 544(7650), 333-336.

Dodd, M.S., Papineau, D., Grenne, T., Slack, J.F., Rittner, M., Pirajno, F., O'Neil, J. \& Little, C.T.S. (2017). Evidence for early life in Earth's oldest hydrothermal vent precipitates. Nature 543(7643), 60-64.

Dong, C., Jin, M., Lingam, M., Airapetian, V.S., Ma, Y. \& van der Holst, B. (2017a). Atmospheric escape from the TRAPPIST-1 planets and implications for habitability. submitted to Proc. Natl. Acad. Sci. USA (arXiv:1705.05535).

Dong, C., Lingam, M., Ma, Y. \& Cohen, O. (2017b). Is Proxima Centauri b habitable? A study of atmospheric loss. Astrophys. J. Lett. 837(2), L26.

Downs, C.J., Hayes, J.P. \& Tracy, C.R. (2008). Scaling metabolic rate with body mass and inverse body temperature: a test of the Arrhenius fractal supply model. Funct. Ecol. 22(2), 239-244.

Dressing, C.D. \& Charbonneau, D. (2015). The occurrence of potentially habitable planets orbiting $M$ dwarfs estimated from the full Kepler dataset and an empirical measurement of the detection sensitivity. Astrophys. J. 807(1), 45.

Ehlmann, B.L. et al. (2016). The sustainability of habitability on terrestrial planets: insights, questions, and needed measurements from Mars for understanding the evolution of Earth-like worlds. J. Geophys. Res. Planets 121(10), 1927-1961.

Enquist, B.J., Economo, E.P., Huxman, T.E., Allen, A.P., Ignace, D.D. \& Gillooly, J.F. (2003). Scaling metabolism from organisms to ecosystems. Nature 423(6940), 639-642.

Forbes, J.C. \& Loeb, A. (2017). Evaporation of planetary atmospheres due to XUV illumination by quasars. submitted to Mon. Not. R. Astron. Soc. (arXiv:1705.06741).

France, K. et al. (2013). The ultraviolet radiation environment around M dwarf exoplanet host stars. Astrophys. J. 763(2), 149.

Frank, A. \& Sullivan, W. (2014). Sustainability and the astrobiological perspective: framing human futures in a planetary context. Anthropocene 5, 32-41.
Fuhrman, J.A., Steele, J.A., Hewson, I., Schwalbach, M.S., Brown, M.V., Green, J.L. \& Brown, J.H. (2008). A latitudinal diversity gradient in planktonic marine bacteria. Proc. Natl. Acad. Sci. USA 105(22), 77747778.

Garraffo, C., Drake, J.J. \& Cohen, O. (2016). The space weather of Proxima Centauri b. Astrophys. J. Lett. 833(1), L4.

Gaston, K.J. (2000). Global patterns in biodiversity. Nature 405(6783), 220-227.

Gaucher, E.A., Thomson, J.M., Burgan, M.F. \& Benner, S.A. (2003). Inferring the palaeoenvironment of ancient bacteria on the basis of resurrected proteins. Nature 425(6955), 285-288.

Gillon, M. et al. (2016). Temperate Earth-sized planets transiting a nearby ultracool dwarf star. Nature 533(7602), 221-224.

Gillon, M. et al. (2017). Seven temperate terrestrial planets around the nearby ultracool dwarf star TRAPPIST-1. Nature 542(7642), 456-460.

Gillooly, J.F., Brown, J.H., West, G.B., Savage, V.M. \& Charnov, E.L. (2001). Effects of size and temperature on metabolic rate. Science $\mathbf{2 9 3}$ (5538), 2248-2251.

Gillooly, J.F., Charnov, E.L., West, G.B., Savage, V.M. \& Brown, J.H. (2002). Effects of size and temperature on developmental time. Nature 417(6884), 70-73.

Gillooly, J.F., Allen, A.P., West, G.B. \& Brown, J.H. (2005). The rate of DNA evolution: effects of body size and temperature on the molecular clock. Proc. Natl. Acad. Sci. USA 102(1), 140-145.

Gillooly, J.F., Allen, A.P., Savage, V.M., Charnov, E.L., West, G.B. \& Brown, J.H. (2006). Response to Clarke and Fraser: effects of temperature on metabolic rate. Funct. Ecol. 20(2), 400-404.

Glazier, D.S. (2015). Is metabolic rate a universal 'pacemaker' for biological processes? Biol. Rev. 90(2), 377-407.

Goldenfeld, N. \& Woese, C. (2011). Life is physics: evolution as a collective phenomenon far from equilibrium. Anпu. Rev. Condens. Matter Phys. 2, 375-399.

Haqq-Misra, J., Kopparapu, R.K. \& Wolf, E.T. (2017). Why do we find ourselves around a yellow star instead of a red star? Int. J. Astrobiol. 1-10. doi:10.1017/S1473550417000118

Heller, R. \& Armstrong, J. (2014). Superhabitable worlds. Astrobiology 14(1), 50-66.

Heller, R. et al. (2014). Formation, habitability, and detection of extrasolar moons. Astrobiology 14(9), 798-835.

Hoehler, T.M. (2007). An energy balance concept for habitability. Astrobiology 7(6), 824-838.

Hooper, D.U. et al. (2005). Effects of biodiversity on ecosystem functioning: a consensus of current knowledge. Ecol. Monogr. 75(1), 3-35.

Horner, J. \& Jones, B.W. (2010). Determining habitability: which exoEarths should we search for life? Int. J. Astrobiol. 9(4), 273-291.

Humphries, M.M. \& McCann, K.S. (2014). Metabolic ecology. J. Anim. Ecol. 83(1), 7-19.

Ives, A.R. \& Carpenter, S.R. (2007). Stability and diversity of ecosystems. Science 317(5834), 58-62.

Johnson, R.E., Combi, M.R., Fox, J.L., Ip, W.-H., Leblanc, F., McGrath, M.A., Shematovich, V.I., Strobel, D.F. \& Waite, J.H. (2008). Exospheres and atmospheric escape. Space Sci. Rev. 139(1), 355-397.

Johnstone, C.P., Güdel, M., Brott, I. \& Lüftinger, T. (2015). Stellar winds on the main-sequence. II. The evolution of rotation and winds. Astron. Astrophys. 577, A28.

Judson, O.P. (2017). The energy expansions of evolution. Nat. Ecol. Evol. 1, 0138. Kasting, J.F. \& Catling, D. (2003). Evolution of a habitable planet. Annu. Rev. Astron. Astrophys. 41(1), 429-463.

Kasting, J.F., Kopparapu, R., Ramirez, R.M. \& Harman, C.E. (2014). Remote life-detection criteria, habitable zone boundaries, and the frequency of Earth-like planets around M and late K stars. Proc. Natl. Acad. Sci. USA 111(35), 12641-12646.

Kauffman, S. (1995). At Home in the Universe: The Search for the Laws of Self-Organization and Complexity. Oxford University Press, Oxford.

Khodachenko, M.L. et al. (2007). Coronal mass ejection (CME) activity of low mass $\mathrm{M}$ stars as an important factor for the habitability of terrestrial exoplanets. I. CME impact on expected magnetospheres of earth-like exoplanets in close-in habitable zones. Astrobiology 7(1), 167-184. 
Kiang, N.Y., Siefert, J., Govindjee \& Blankenship, R.E. (2007). Spectral signatures of photosynthesis. I. review of earth organisms. Astrobiology $7(1), 222-251$.

Kingsolver, J.G. (2009). The well-temperatured biologist. Am. Nat. 174(6), $755-768$.

Kingsolver, J.G. \& Huey, R.B. (2008). Size, temperature, and fitness: three rules. Evol. Ecol. Res. 10(2), 251-268.

Kopparapu, R.K., Ramirez, R., Kasting, J.F., Eymet, V., Robinson, T.D., Mahadevan, S., Terrien, R.C., Domagal-Goldman, S., Meadows, V. \& Deshpande, R. (2013). Habitable zones around main-sequence stars: new estimates. Astrophys. J. 765(2), 131.

Lammer, H. (2013). Origin and Evolution of Planetary Atmospheres: Implications for Habitability. Springer Briefs in Astronomy. Springer, Berlin.

Lammer, H. et al. (2009). What makes a planet habitable? Astron. Astrophys. Rev. 17(2), 181-249.

Levin, S.A. (1998). Ecosystems and the biosphere as complex adaptive systems. Ecosystems 1(5), 431-436.

Levins, R. (1968). Evolution in Changing Environments: Some Theoretical Explorations. Princeton University Press, Princeton.

Levins, R. (1969). Some demographic and genetic consequences of environmental heterogeneity for biological control. Bull. Entomol. Soc. Am. 15(3), 237-240.

Lineweaver, C.H. \& Chopra, A. (2012). The habitability of our earth and other earths: astrophysical, geochemical, geophysical, and biological limits on planet habitability. Annu. Rev. Earth Planet. Sci. 40, 597-623.

Lineweaver, C.H. \& Davis, T.M. (2002). Does the rapid appearance of life on earth suggest that life is common in the universe? Astrobiology 2(3), 293-304.

Lingam, M. (2016). Interstellar travel and galactic colonization: insights from percolation theory and the Yule process. Astrobiology 16(6), 418-426.

Lingam, M. \& Loeb, A. (2017). Enhanced interplanetary panspermia in the TRAPPIST-1 system. Proc. Natl. Acad. Sci. USA (in press). doi:10.1073/ pnas. 1703517114.

Loeb, A., Batista, R.A. \& Sloan, D. (2016). Relative likelihood for life as a function of cosmic time. J. Cosmol. Astropart. Phys. 8, 040.

Lovelock, J.E. \& Margulis, L. (1974). Atmospheric homeostasis by and for the biosphere: the Gaia hypothesis. Tellus 26(1-2), 2-10.

Luger, R. \& Barnes, R. (2015). Extreme water loss and abiotic $\mathrm{O}_{2}$ buildup on planets throughout the habitable zones of M dwarfs. Astrobiology 15(2), 119-143.

Marquet, P.A., Quiñones, R.A., Abades, S., Labra, F., Tognelli, M., Arim, M. \& Rivadeneira, M. (2005). Scaling and power-laws in ecological systems. J. Exp. Biol. 208(9), 1749-1769.

Martin, W., Baross, J., Kelley, D. \& Russell, M.J. (2008). Hydrothermal vents and the origin of life. Nat. Rev. Microbiol. 6(11), 805-814.

Martinez, C.L.F. (2014). SETI in the light of cosmic convergent evolution. Acta Astron. 104(1), 341-349.

Mayhew, P.J., Bell, M.A., Benton, T.G. \& McGowan, A.J. (2012). Biodiversity tracks temperature over time. Proc. Natl. Acad. Sci. USA 109(38), 15141-15145.

McKay, C.P. (2014). Requirements and limits for life in the context of exoplanets. Proc. Natl. Acad. Sci. USA 111(35), 12628-12633.

Miller, S.L. \& Lazcano, A. (1995). The origin of life-did it occur at high temperatures? J. Mol. Evol. 41(6), 689-692.

Moles, A.T. et al. (2014). Which is a better predictor of plant traits: temperature or precipitation? J. Veg. Sci. 25(5), 1167-1180.

Monod, J. (1971). Chance and Necessity: An Essay on the Natural Philosophy of Modern Biology. Alfred A. Knopf, New York.

Morowitz, H. \& Smith, E. (2007). Energy flow and the organization of life. Complexity 13(1), 51-59.

Morris, S.C. (2003). Life's Solution: Inevitable Humans in a Lonely Universe. Cambridge University Press, Cambridge.

Owen, J.E. \& Alvarez, M.A. (2016). UV driven evaporation of close-in planets: energy-limited, recombination-limited, and photon-limited flows. Astrophys. J. 816(1), 34.

Pace, N.R. (1991). Origin of life-facing up to the physical setting. Cell 65(4), $531-533$.
Pace, N.R. (2001). The universal nature of biochemistry. Proc. Natl. Acad. Sci. USA 98(3), 805-808.

Pascal, R., Pross, A. \& Sutherland, J.D. (2013). Towards an evolutionary theory of the origin of life based on kinetics and thermodynamics. Open Biol. 3(11), 130156.

Picard, A. \& Daniel, I. (2013). Pressure as an environmental parameter for microbial life - a review. Biophys. Chem. 183, 30-41.

Price, C.A. et al. (2012). Testing the metabolic theory of ecology. Ecol. Lett. 15(12), 1465-1474.

Purvis, A. \& Hector, A. (2000). Getting the measure of biodiversity. Nature 405(6783), 212-219.

Ramirez, R.M. \& Kaltenegger, L. (2014). The habitable zones of premain-sequence stars. Astrophys. J. Lett. 797(2), L25.

Ranjan, S. \& Sasselov, D.D. (2016). Influence of the UV environment on the synthesis of prebiotic molecules. Astrobiology 16(1), 68-88.

Ribas, I. et al. (2016). The habitability of Proxima Centauri b. I. Irradiation, rotation and volatile inventory from formation to the present. Astron. Astrophys. 596, A111.

Rogers, L.A. (2015). Most 1.6 earth-radius planets are not rocky. Astrophys. J. 801(1), 41.

Rosenzweig, M.L. (1995). Species Diversity in Space and Time. Cambridge Univ. Press, Cambridge.

Rothschild, L.J. \& Mancinelli, R.L. (2001). Life in extreme environments. Nature 409(6823), 1092-1101.

Rushby, A.J., Claire, M.W., Osborn, H. \& Watson, A.J. (2013). Habitable zone lifetimes of exoplanets around main sequence stars. Astrobiology 13(9), 833-849

Savage, V.M., Gillooly, J.F., Brown, J.H., West, G.B. \& Charnov, E.L. (2004). Effects of body size and temperature on population growth. Am. Nat. 163(3), 429-441.

Savage, V.M., Deeds, E.J. \& Fontana, W. (2008). Sizing up allometric scaling theory. PLoS Comput. Biol. 4(9), e1000171.

Scalo, J. et al. (2007). M stars as targets for terrestrial exoplanet searches and biosignature detection. Astrobiology 7(1), 85-166.

Scharf, C. \& Cronin, L. (2016). Quantifying the origins of life on a planetary scale. Proc. Natl. Acad. Sci. USA 113(29), 8127-8132.

Schulte, P.M. (2015). The effects of temperature on aerobic metabolism: towards a mechanistic understanding of the responses of ectotherms to a changing environment. J. Exp. Biol. 218(12), 1856-1866.

Schulze-Makuch, D. \& Guinan, E. (2016). Another Earth 2.0? Not so fast. Astrobiology 16(11), 817-821.

Schulze-Makuch, D. \& Irwin, L.N. (2008). Life in the Universe: Expectations and Constraints. Springer, Berlin.

Seager, S. (2010). Exoplanet Atmospheres: Physical Processes. Princeton Series in Astrophysics. Princeton University Press, Princeton.

Shields, A.L., Ballard, S. \& Johnson, J.A. (2016). The habitability of planets orbiting M-dwarf stars. Phys. Rep. 663(1), 1-38.

Simpson, G.G. (1964). The nonprevalence of humanoids. Science 143(3608), 769-775.

Spiegel, D.S. \& Turner, E.L. (2012). Bayesian analysis of the astrobiological implications of life's early emergence on Earth. Proc. Natl. Acad. Sci. USA 109(2), 395-400.

Tarter, J.C. et al. (2007). A reappraisal of the habitability of planets around M dwarf stars. Astrobiology 7(1), 30-65.

Tasker, E. et al. (2017). The language of exoplanet ranking metrics needs to change. Nat. Astron. 1, 0042.

Thompson, D.W. (1942). On Growth and Form. Cambridge University Press, Cambridge.

Tian, F. \& Ida, S. (2015). Water contents of Earth-mass planets around M dwarfs. Nat. Geosci. 8(3), 177-180.

Valencia, D., O'Connell, R.J. \& Sasselov, D. (2006). Internal structure of massive terrestrial planets. Icarus 181(2), 545-554.

Vidotto, A.A., Jardine, M., Morin, J., Donati, J.-F., Lang, P. \& Russell, A.J. B. (2013). Effects of M dwarf magnetic fields on potentially habitable planets. Astron. Astrophys. 557, A67.

Vladilo, G., Murante, G., Silva, L., Provenzale, A., Ferri, G. \& Ragazzini, G. (2013). The habitable zone of earth-like planets with different levels of atmospheric pressure. Astrophys. J. 767(1), 65. 
Wang, Z., Brown, J.H., Tang, Z. \& Fang, J. (2009). Temperature dependence, spatial scale, and tree species diversity in eastern Asia and North America. Proc. Natl. Acad. Sci. USA 106(32), 1338813392 .

Ward, P. \& Brownlee, D. (2000). Rare Earth: Why Complex Life Is Uncommon in the Universe. Copernicus, New York.

Weiss, M.C., Sousa, F.L., Mrnjavac, N., Neukirchen, S., Roettger, M., Nelson-Sathi, S. \& Martin, W.F. (2016). The physiology and habitat of the last universal common ancestor. Nat. Microbiol. 1, 16116.

West, G.B., Brown, J.H. \& Enquist, B.J. (2001). A general model for ontogenetic growth. Nature 413(6856), 628-631.

Winn, J.N. \& Fabrycky, D.C. (2015). The occurrence and architecture of exoplanetary systems. Annu. Rev. Astron. Astrophys. 53, 409-447.
Wolf, E.T. (2017). Assessing the habitability of the TRAPPIST-1 system using a 3D climate model. Astrophys. J. Lett. 839(1), L1.

Wood, B.E., Linsky, J.L., Müller, H.-R. \& Zank, G.P. (2001). Observational estimates for the mass-loss rates of $\alpha$ Centauri and Proxima Centauri Using Hubble space telescope Lyo spectra. Astrophys. J. Lett. 547(1), L49-L52.

Wood, B.E., Müller, H.-R., Zank, G.P. \& Linsky, J.L. (2002). Measured mass-loss rates of solar-like stars as a function of age and activity. Astrophys. J. 574(1), 412-425.

Zendejas, J., Segura, A. \& Raga, A.C. (2010). Atmospheric mass loss by stellar wind from planets around main sequence M stars. Icarus 210(2), 539-544.

Zeng, L., Sasselov, D.D. \& Jacobsen, S.B. (2016). Mass-radius relation for rocky planets based on PREM. Astrophys. J. 819(2), 127. 